\title{
Hygiène et assainissement : étude multicentrique contribuant à améliorer leur qualité dans la maternité de deux centres de santé communautaire et universitaire (Banconi et Konobougou), Mali
}

\section{Hygiene and sanitation: multicenter study helping to improve their quality in maternity two community and university health centers (Banconi and Konobougou), Mali}

\author{
Kané $\mathrm{AM}^{1}$, Sidibé $\mathrm{BM}^{2}$, Sidibé $\mathrm{S}^{2}$, Coulibaly $\mathrm{MB}^{1 *}$, Barry $\mathrm{K}^{3}$, Touré $\mathrm{M}^{4}$, Dicko Traoré $\mathrm{F}^{3,5}$
}

1. Centre de santé communautaire et universitaire de Konobougou (Ségou-Mali)

2. Centre de santé communautaire et universitaire de Banconi (Bamako-Mali)

3. Département de médecine de famille/médecine communautaire FMOS, Mali

4. Service de gynécologie du CHU de l'hôpital du Mal

5. Département de pédiatrie du CHU Gabriel Touré, Mali

*Auteur correspondant : Dr Mamadou Bayo COULIBALY, spécialiste en médecine de famille/médecine communautaire, Directeur technique du CSCom U de Konobougou, Chargé d'encadrement clinique D.E.S médecine de famille/médecine communautaire (Mali). bayo_coul@yahoo.fr ; Cel : 0022376067882

\section{Résumé}

Introduction : Au Mali, il ressort de l'analyse de la situation de base en matière de santé et environnement une insuffisance de l'hygiène et de l'assainissement en milieu hospitalier. L'objectif de cette étude était d'évaluer la qualité de l'hygiène et de l'assainissement à la maternité du centre de santé communautaire et universitaire de Banconi et de Konobougou afin de contribuer à leur amélioration. Matériel and méthodes : II s'agissait d'une étude de recherche action de Susmam multicentrique réalisée dans la maternité des centres de santé communautaire et universitaire : Banconi et Konobougou. Elle a couvert la période de 1er mai au 31 août 2019. Ont été inclus dans l'étude les membres des associations de santé communautaires (ASACO) et le personnel des maternités consentants. Résultats : Après la mise en place du plan d'action ente l'évaluation initiale et finale ; le lavage des mains entre deux malades par le personnel soignant a évolué de $25 \%$ à $100 \%$ à Konobougou et de $66,67 \%$ à $100 \%$ à Banconi. En outre, en moyenne $55 \%$ du personnel savaient préparer l'eau de décontamination contre $90 \%$ au terme de l'étude à Konobougou alors qu'à Banconi cela a évolué de $76,33 \%$ à $100 \%$, après le plan d'action. Le tri des déchets était effectué par tout le monde et un vidange quotidien des poubelles respecté par les manœuvres. Conclusion : La connaissance des mornes sur l'hygiène et l'assainissement et sa mise en œuvre correcte permettent de réduire le risque des infections nosocomiales.

Mots clés : Hygiène, assainissement, maternité, Banconi, Konobougou, Mali

\footnotetext{
Abstract

Introduction: In Mali, an analysis of the basic health and environment situation shows that there is insufficient hygiene and sanitation in hospitals. The objective of this study was to assess the quality of hygiene and sanitation
}

at the maternity unit of the community and university health center of Banconi an urban center at Bamako and Konobougou a rural center in Ségion région in, order to contribute to their improvement. Method and materials: This was a multicentre Susmam action research study carried out in the maternity unit of community and university health centers: Banconi and Konobougou. It covered the period from May 1 to August 31, 2019. The members of community health associations (ASACO) and the staff of consenting maternities were included in the study. Results: After the implementation of the action plan between the initial and final evaluation; hand washing between two patients by nursing staff has increased from $25 \%$ to $100 \%$ in Konobougou and that of Banconi from $66.67 \%$ to $100 \%$. In addition, on average $55 \%$ of the staff knew how to prepare the decontamination water against $90 \%$ at the end of the study in Konobougou while in Banconi this changed from $76.33 \%$ to $100 \%$ after the action plan. The sorting of waste was carried out by everyone and a daily emptying of the bins respected by the laborers. Conclusion: Knowledge of dreary hygiene and sanitation and its correct implementation can reduce the risk of nosocomial infections.

Keywords: Hygiene, sanitation, Maternity, Banconi, Konobougou, Mali

\section{INTRODUCTION}

Depuis ses débuts au XIXème siècle, l'approche de l'hygiène hospitalière s'est inversée. Alors que Semmelweis, le père de la désinfection des mains, fut banni par ses confrères et mourut misérablement, la communauté scientifique, aujourd'hui, sous la contrainte de l'extension de la menace infectieuse nosocomiale, multiplie les recommandations à l'adresse du personnel hospitalier (1). M. MAISONNET (1966) définit l'hygiène hospitalière comme "une discipline qui traite aussi bien, des multiples problèmes posés par la prévention de l'infection et de la surinfection à l'hôpital, que des aspects sociaux et moraux qui découlent de toute hospitalisation» (2). Peu d'études portent sur LA REPRESENTATION DE L'HYGIENE. Zérillo (1998) interroge des étudiants (en psychologie et en formation d'infirmière) et montre que la représentation de l'hygiène s'articule autour des éléments " prévention des maladies », " bien être », « respect des autres », et " éviter les mauvaises odeurs » (3). La contamination de l'environnement (eau, air, surfaces) peut être à l'origine des infections. Ainsi, il est important de développer un système de surveillance de la contamination de l'environnement lorsque celui-ci est 
maîtrisé. Préalablement, il faut définir et classer les zones en fonction des risques (faible, modéré, haut et très haut risque) en accord avec les guides ou les recommandations quand ceux-ci existent (4).

Dans toutes les régions du monde, les hôpitaux offrent un écosystème propice à la diffusion d'agents infectieux, potentiellement épidémiogènes, et à l'acquisition de résistances microbiennes. Les risques infectieux au sein des hôpitaux sont multiples. On distingue notamment les infections nosocomiales, les risques épidémiques et les risques infectieux professionnels (5). En Afrique, la maîtrise du risque infectieux en milieu hospitalier reste une question cruciale. Bien souvent, les analyses se limitent à une évaluation des décalages entre des normes techniques et des pratiques hospitalières. L'insuffisance des connaissances théoriques est régulièrement invoquée, conduisant à un cycle de formation-évaluation qui semble largement voué à l'échec (5).

Le respect des protocoles d'hygiène est souvent presenté comme synonyme d'asepsie relationnelle : le port des gants, masques et sur-blouses constituant autant d'ecrans dans la relation et de sources potentielles d'anxiété pour le malade ». Mais peut-on en conclure simplement que les pratiques d'hygiène se présentent comme une "construction de compromis entre des exigences contradictoires »(1). Aussi, les hôpitaux doivent-ils être considérés comme des cibles de l'action de santé publique. La mise en place de pratiques d'hygiène médicale rigoureuses tout autant que diverses mesures de prévention (notamment vaccinales) participent de la lutte contre les risques infectieux hospitaliers et se sont assez largement généralisées dans les hôpitaux des pays du Nord au cours des dernières décennies (5).

L'hygiène hospitalière ne peut se résumer à la lutte contre les infections acquises à l'hôpital, ni aux seules études de l'environnement hospitalier. Elle doit aussi concerner l'organisation des soins et des pratiques, en étudier l'efficacité et l'efficience et valider leur pertinence dans l'objectif d'une meilleure sécurité. Elle est un des piliers de la qualité en milieu de soins ; à cet égard elle est multidisplinaire (6).

Au Mali, il ressort de l'analyse de la situation de base en matière de santé et environnement, effectuée en 2011 par les acteurs de santé, l'insuffisance d'adoption de bonnes pratiques d'hygiènes, de sa promotion et de reconnaissance des comportements à risques (précautions universelles : mesures individuelles et collectives, etc.). II a été aussi noté le manque de dispositif de lavage des mains, de solution hydro alcoolique, de réflexe de lavage, d'organisation et de fonctionnement de comités d'hygiène et d'assainissement. C'est ainsi que nous avons mené ce travail multicentrique pour évaluer la qualité de l'hygiène et de l'assainissement à la maternité du CSCom universitaire de Banconi et de Konobougou, respectivement urbain et rural, afin de contribuer à leur amélioration.

\section{MATERIEL ET METHODES}

II s'agissait d'une étude de recherche action de Susmam (7) multicentrique réalisée dans la maternité des centres de santé communautaire et universitaire : l'une urbaine (Banconi) et l'autre rurale (Konobougou). Elle a couvert une période de quatre mois, allant de 1er mai au 31 août 2019.

Ont été inclus dans l'étude les membres des associations de santé communautaires (ASACO) et le personnel des maternités consentants. N'ont pas été introduit dans cette étude l'ensemble du personnel du centre ne travaillant pas dans les maternités et tout autre membre de l'ASACO et personnel de la maternité non consentants.

Une fiche d'enquête a été soumise auprès du personnel et les membres de l'ASACO. En plus, nous avons observé le personnel dans centaines tâches. Une autre grille a été introduite pour l'évaluation de l'environnement et des matériels d'hygiène assainissement. Les données ont été analysées par Epi info 7 version 3.5.3.

Les variables étudiées étaient : l'existence de poubelles, l'existence d'affiches indiquant les catégories de déchets, le respect des consignes de tri à la source, le respect de la périodicité d'enlèvement des déchets, le nettoyage régulier des contenants, l'état des contenants, l'état du dépôt transitoire, la disponibilité et l'état des équipements de protection, le port des équipements de protection, l'existence d'aires de lavage des mains et de matériels nécessaires pour le lavage des mains, le lavage des mains aux différents moments, le nettoyage régulier des salles, la stérilisation des matériaux, les définitions opératoires et les risques sanitaires et environnementaux. Dans un premier temps nous avons fait une évaluation initiale afin de connaitre les points forts, les insuffisances et les goulots d'étranglements. Dans un deuxième temps, nous avons mis en œuvre le plan d'action que nous avons élaboré avec le personnel et les membres de l'ASACO après une formation et sensibilisation des différents intervenants. Enfin, une évaluation finale a été réalisée pour voir l'effet du plan d'action.

L'étude s'est déroulée en respectant les règles d'éthiques liées à la recherche sur les sujets humains en vigueur. II ne s'agissait pas d'une étude expérimentale sur l'Homme. II s'agissait d'une étude permettant d'apporter des solutions pour résoudre les problématiques de l'hygiène et l'assainissement. Un consentement écrit et signé a été obtenu du directeur technique du centre, de la responsable de la maternité et du président de l'ASACO. L'étude ne comportait aucun risque additionnel chez les personnes. Les noms et prénoms des sujets n'ont pas été utilisés. Les données étaient gardées de façon confidentielle.

\section{RESULTATS :}

Dans cette étude, nous avons introduit 39 personnes à l'évaluation initiale et finale.

La première évaluation réalisée en Mai a permis d'identifier les insuffisances et la deuxième de vérifier l'action positive et/ou négative des changements 
apportés. Pour un aperçu nous avons choisi de faire une comparaison par grande ligne sur les deux résultats.

Les matrones ont représenté la majorité du personnel de l'unité de maternité avec $46 \%$ (soit $n=11$ ) à Konobougou alors que les infirmières obstétriciennes ont représenté $53,33 \%(n=8)$ (figure I).

Les deux centres n'ont jamais été en pénurie d'eau à cause de leur dotation en eau (de SOMAGEP qui alimente également une Cuve de stockage d'eau pour Banconi ; SOMAGEP et deux forages pour Konobougou toutes fonctionnelles), capitale pour assurer l'hygiène et l'assainissement.

Au début de l'enquête nous avons eu $34,78 \%$ de personnel respectant le lavage des mains à la prise de service contre $78,26 \%$ à la fin de l'étude à Konobougou et de 26,67 \% à 93, 33 \% pour Banconi (tableaux I et II).

Le lavage des mains entre deux malades n'était pas respecté par $25 \%$ du personnel soignant qui sont parvenus à respecter ce moment (100\%) à Konobougou et celui de Banconi a évolué de $66,67 \%$ à $100 \%$.

Nous avons constaté une évolution de l'évaluation initiale à l'évaluation finale le lavage des mains avant la CPN ou l'accouchement de $40 \%$ à $85 \%$ et de $46,67 \%$ à $86,67 \%$ respectivement pour Konobougou et Banconi.

Les trois couleurs de poubelle (Rouge, Jaune, Noire) et les boites de sécurité existaient au niveau de la maternité et étaient toutes en bon état et utilisées par tout le personnel dans les deux centres. Le tri des déchets était effectué partout et un vidage quotidien des poubelles respecté par les manœuvres.

Le nettoyage de sol était effectué une fois par jour. Ailleurs, il était réalisé en cas de besoin, dans la salle d'accouchement, selon les normes d'hygiène et d'assainissement dans les deux centres. II existait l'eau de décontamination dans la salle d'accouchement et bien conservée par l'équipe de garde.

En outre, en moyenne onze personnes sur vingt soit $55 \%$ savaient préparer l'eau de décontamination contre $90 \%$ au terme de l'étude à Konobougou, alors qu'à Banconi cela a évolué de $76,33 \%$ à $100 \%$. Dans la majorité des cas, le renouvellement d'eau de décontamination était effectué une fois/jour et si besoin en fin de l'étude (tableaux III et $\mathrm{VI})$.

Seule une technicienne de surface ne portait toujours pas la tenue, cela relevait de l'ASACO qui avait passé la commande mais non livrée au cours de la période d'étude après le plan d'action à Konobougou, tandis que $100 \%$ du personnel portaient la blouse en bon état à Banconi pendant les deux évaluations.

Le port de bonnet était respecté dans les $78,26 \%$ et 13,33 $\%$ au début contre $95,65 \%$ et $60 \%$ à la fin d'étude respectivement à Konobougou et Banconi, parmi lesquels les mauvais bonnets (sales ou mal adaptés) ont représenté $27,78 \%$ au début et $4,55 \%$ à la fin à Konobougou et ils étaient tous en bon état à Banconi. La bavette était portée à $21,74 \%(n=5)$ et $20 \%$ contre $100 \%$ et $66,67 \%$ à la fin de l'étude à Konobougou et à Banconi. Elles étaient toutes en bon état (non déchirées, bien adaptées et propres) Seulement 8,70\% de personnel portaient des lunettes contre $100 \%$ à la fin de l'étude à Konobougou. Aucun personnel technique ne portait de lunette lors des accouchements, cela a été amélioré à 60 $\%$ après l'action. Les lunettes étaient disponibles et en bon état à la fin. En moyenne, $55 \%$ d'agents portaient le tablier contre $85 \%$ au terme de l'étude. Au début de l'enquête, deux tabliers sur les onze portés $(18,18 \%)$ ont été réutilisés avant le nettoyage et $100 \%$ des dix sept tabliers portés étaient en bon état à la dernière évaluation. La totalité du personnel technique portait des gants lors des CPN, CPON et accouchements.

II existait un stérilisateur fonctionnel durant la période de l'étude dans les deux centres. Le temps de stérilisation n'avait pas été respecté à $15 \%$ et respecté plus tard par tous les agents (100\%) à Konobougou et $100 \%$ dans les deux évaluations à Banconi. La gestion des matériels (décontamination, stérilisation et conservation) a été améliorée de $56,52 \%$ et $80 \%$ à $82,61 \%$ et $100 \%$ respectivement à Konobougou et à Banconi.

A la fin de notre travail, tout le personnel de la maternité avait reçu une formation sur l'hygiène et l'assainissement, maitrisait la technique de tri de déchets, connaissait les étapes et les moments de lavage des mains.

\section{DISCUSSION :}

La première évaluation réalisée en Mai a permis d'identifier les insuffisances et la deuxième de vérifier l'action positive et/ou négative des changements apportés. Pour un aperçu, nous avons choisi de faire une comparaison par grande ligne sur les deux résultats.

Les matrones ont représenté la majorité du personnel de l'unité de maternité avec $46 \%$ (soit $n=11$ ) à Konobougou alors que les infirmières obstétriciennes ont représenté $53,33 \%(n=8)$

Les deux centres n'ont jamais été en pénurie d'eau à cause de leur dotation en eau (de SOMAGEP qui alimente également une Cuve de stockage d'eau pour Banconi ; SOMAGEP et deux forages pour Konobougou toutes fonctionnelles), capitale pour assurer l'hygiène et l'assainissement.

Au début de l'enquête, nous avons eu $34,78 \%$ de personnel respectant le lavage des mains à la prise de service contre $78,26 \%$ à la fin de l'étude à Konobougou et de $26,67 \%$ à $93,33 \%$ pour Banconi. Ces taux sont supérieurs à celui de SIDIBE DM qui avait obtenu respectivement $15 \%$ et $76,16 \%$ (8).

Le lavage des mains entre deux malades n'était pas respecté par $25 \%$ du personnel soignant qui sont parvenus à respecter ce moment (100\%) à Konobougou et celui de Banconi a évolué de 66,67 \% à $100 \%$ contre $94,44 \%$ obtenu par COULIBALY KB à la fin de l'étude (9). Une habitude qui réduirait le risque de transmission des infections nosocomiales

Nous avons constaté une évolution, de l'évaluation initiale à l'évaluation finale, du lavage des mains avant la CPN ou l'accouchement de $40 \%$ à $85 \%$ et de $46,67 \%$ à $86,67 \%$ 
respectivement pour Konobougou et Banconi. Cela nécessitera un suivi régulier.

Alors que, selon C. Brun-Buisson, la mise à disposition de solution hydro alcoolique augmentait l'observance de l'hygiène des mains de $42 \%$ à $61 \%$ (10). Pour M Schindler, une réduction significative des infections nosocomiales a été ainsi obtenue mais, depuis ce premier succès, les taux d'observance de l'hygiène des mains par le personnel n'augmentent pas suffisamment. II a trouvé que les soignants se désinfectent les mains sensiblement plus après avoir été en contact avec un malade (70 \%) qu'avant d'entrer en contact avec lui (40\%) (1). Tandis que $\mathrm{F}$ Traore Dicko et al ont trouvé que le personnel semble avoir une bonne connaissance théorique $(87,2$ à $96,4 \%$ ) des mesures d'hygiène des mains à adopter avant et après les gestes les plus courants dans l'unité. II y'a une inobservance de l'hygiène des mains dans $33,3 \%$ des cas (11). De nombreuses autres études ont montré que le taux de "compliance " au lavage des mains avec de l'eau et du savon était de moins de $50 \%(2,12,13)$.

Les trois couleurs de poubelle (Rouge, Jaune, Noire) et les boites de sécurité existaient au niveau de la maternité et étaient toutes en bon état et utilisées par tout le personnel dans les deux centres. Le tri des déchets était effectué partout et un vidage quotidien des poubelles respecté par les manœuvres. Le tri des DBM était inadapté dans $53,5 \%$ des services et l'utilisation du système de codage par couleur effective dans $31,4 \%$ des services ; des boîtes de sécurité étaient disponibles dans $82,5 \%$ et leur utilisation effective dans $51,1 \%$ d'entre eux (14). Une faible proportion des agents utilisait les sacs poubelles $(36,36 \%)$ et les conteneurs $(11,54 \%)(15)$.

Le nettoyage du sol était effectué une fois par jour. Par ailleurs, il était réalisé en cas de besoin, dans la salle d'accouchement, selon les normes d'hygiène et d'assainissement dans les deux centres. II existait l'eau de décontamination dans la salle d'accouchement et bien conservée par l'équipe de garde. Tous ceux-ci ont été pérennisés grâce à la surveillance du comité d'hygiène et d'assainissement et le Directeur Technique du CSCom. Tandis que pour $\mathrm{G}$. Clapeau la fréquence de nettoyage et/ou de la désinfection est journalière pour les sols dans $59 \%$ des structures enquêtées et la fréquence de traitement des instruments quels qu'ils soient après chaque acte est bien respecté dans tous les centres (16). En outre, en moyenne onze personnes sur vingt soit $55 \%$ savaient préparer l'eau de décontamination contre $90 \%$ au terme de l'étude à Konobougou, alors qu'à Banconi cela à évolué de $76,33 \%$ à $100 \%$. Dans la majorité des cas, le renouvellement d'eau de décontamination était effectué une fois/jour et si besoin en fin de l'étude. Cette préparation et son renouvellement ont été améliorés après un rappel et un suivi au cours de la période d'étude et nécessite toujours une continuité de formation. Le nettoyage des toilettes est bihebdomadaire, d'où l'état non satisfaisant des toilettes entre les nettoyages.

Seule une technicienne de surface ne portait toujours pas la ténue, cela relevait de l'ASACO qui avait passé la commande mais non livrée au cours de la période d'étude. Après le plan d'action à Konobougou, tandis que $100 \%$ du personnel portaient la blouse en bon état, à Banconi pendant les deux évaluations seulement quatre personnes soient $18,18 \%$ n'avaient pas une blouse en bon état contre $90,91 \%$ de bon état après un plan d'action à Konobougou. Selon Duroy E. et al $50 \%$ des externes portaient leurs blouses trop grandes (17). Dans l'étude de Yao-Bathaix, le port des blouses était à $97,6 \%$ (18).

Le port de bonnet était respecté dans les $78,26 \%$ et 13,33 $\%$ au début contre $95,65 \%$ et $60 \%$ à la fin d'étude respectivement à Konobougou et Banconi, parmi lesquels les mauvais bonnets (sales ou mal adaptés) ont représenté $27,78 \%$ au début et $4,55 \%$ à la fin à Konobougou, et ils étaient tous en bon état à Banconi. La bavette était portée à $21,74 \%(n=5)$ et $20 \%$ contre $100 \%$ et $66,67 \%$ à la fin de l'étude à Konobougou et à Banconi. Elles étaient toutes en bon état (non déchirées, bien adaptées et propres) Seulement 8,70\% du personnel portaient des lunettes contre $100 \%$ à la fin de l'étude à Konobougou. Aucun personnel technique ne portait de lunette lors des accouchements, cela a été amélioré à 60 $\%$ après l'action. Les lunettes étaient disponibles et en bon état à la fin. En moyenne, $55 \%$ d'agents portaient le tablier contre $85 \%$ au terme de l'étude. Au début de l'enquête, deux tabliers sur les onze portés $(18,18 \%)$ ont été réutilisés avant le nettoyage et $100 \%$ des dix sept tabliers portés étaient en bon état à la dernière évaluation. La totalité du personnel technique portait des gants lors des $\mathrm{CPN}, \mathrm{CPON}$ et accouchements. Ces résultats sur l'usage des équipements de protection individuelle sont salutaires et permettent aux praticiens d'éviter les infections nosocomiales au cours de l'exercice de leurs fonctions. Notre contribution a amené les $17,39 \%$ de port de bottes à $91,30 \%$ à Konobougou. Selon Bengaly $L$ et al, le taux global de port de gants était de $23,30 \%$ et $27,20 \%$ respectivement avant et après intervention (19). Tandis que Yao-Bathaix M F avait trouvé pour le port de gants à usage unique à $71,5 \%$ qui étaient les moyens de protection individuelle les plus utilisés (15). Les gants représentent une barrière efficace contre la transmission croisée des germes, en particulier lors d'épidémies ; ils réduisent le niveau de contamination des mains par la flore acquise au cours des soins ; le port des gants est largement répandu lors des activités de soins, mais pas toujours dans des situations où il est indiqué (20). Pour porter les équipements de protection individuelles, il faut une bonne disponibilité alors que dans l'étude de $\mathrm{M}$ Ndiaye et al et de Chevalier B et al, elle était à $45,3 \%$ dans les deux cas $(14,21)$

II existait un stérilisateur fonctionnel durant la période de l'étude dans les deux centres. Le temps de stérilisation n'avait pas été respecté à $15 \%$ et respecté plus tard par tous les agents (100\%) à Konobougou et $100 \%$ dans les deux évaluations à Banconi. La gestion de matériels (décontamination, stérilisation et conservation) a été améliorée de $56,52 \%$ et $80 \%$ à $82,61 \%$ et $100 \%$, 
respectivement à Konobougou et à Banconi. Ce qui diminuerait le risque d'infection nosocomiale.

A la fin de notre travail, tout le personnel des deux maternités avait reçu une formation sur l'hygiène et l'assainissement, maitrisait la technique de tri de déchets, connaissait les étapes et les moments de lavage des mains. Ce qui réduirait considérablement le risque des infections nosocomiales.

Ce travail a été rendu possible grâce : à l'engagement du personnel et les affiches d'aide mémoire sur le lavage des mains et la gestion des déchets biomédicaux dans la salle d'accouchement ; à l'implication de l'ASACO en dotation de matériels (nécessaires pour l'hygiène des mains, le nettoyage du local et des matériels et pour le tri des déchets) et au suivi du comité d'hygiène et d'assainissement du CSCom. Les fiches de protocoles de soins sont connues par 54,5\%(1 44/264) des personnes interrogées et un enseignement d'hygiène a été suivi par $52,2 \%(138 / 264$ soignants) (21). Selon Duroy, E, la formation en hygiène hospitalière est un élément essentiel de la prévention des infections nosocomiales et de la qualité des soins (17). Pour Rogues MA, la formation et l'information en matière d'hygiène hospitalière s'inscrit dans la politique d'amélioration de la sécurité et de la qualité des soins (2).

Notre étude de recherche action portait des points forts et des points à améliorer :

$>$ Les points forts de notre recherche :

- La disponibilité et la participation active du personnel de la maternité et les membres de l'ASACO dans l'élaboration et la mise en œuvre du plan d'action ;

- L'implication du comté d'hygiène et assainissement du CSCom-U de Konobougou.

$>$ Les points à améliorer :

- Le changement de comportement du personnel (par rapport au lavage des mains, port des équipements de protection, tri des déchets biomédicaux, gestion du matériel et à l'assainissement du local) ;

- Le suivi au niveau de la maternité

\section{CONCLUSION}

La connaissance des mornes sur l'hygiène et l'assainissement et sa mise en œuvre correcte permettent de réduire le risque des infections nosocomiales. Un suivi continuel de la qualité de l'hygiène dans les services contribue largement à la lutte contre les infections nosocomiales. Ces infections constituent une inquiétude pour le personnel et pour les malades. Elles constituent une cause de morbidité et de mortalité avec une perte de ressources pour la structure de santé et une baisse du revenu pour les ménages.

\section{RECOMMANDATIONS}

Bien que les améliorations aient été constatées dans tous les domaines de l'hygiène et l'assainissement, ces améliorations doivent être soutenues. Pour cela, à la lumière de cette étude, nous avons recommandé :

- La formation adaptée et continue des professionnels de santé et les membres de l'ASACO, sur les modules de l'hygiène et l'assainissement ;

- Une supervision interne à la maternité par le Directeur technique du centre et les responsables du comité d'hygiène et d'assainissement.

\section{REFERENCES}

1. Schindler M: Avoir raison de la souillure : l'observance de l'hygiène des mains en milieu hospitalier. Sciences sociales et santé. 2013 ; 4(31) : 5-28.

2. Rogues MA Hajjar J : L'équipe opérationnelle d'hygiène hospitalière et de prévention des infections nosocomiales dans les établissements de santé; HygieneS. 2001 ; IX (6) : 380-382.

3. Morlot $\mathbf{R}$, Salès-Wuillemin E: Effet des pratiques et des connaissances sur la représentation sociale d'un objet : application à l'hygiène hospitalière. Revue internationale de psychologie sociale. 2008 ; 4 (21) : 89-114.

4. Brocard-Lemort C: Normes et recommandations en hygiène environnementale hospitalière. Annales de Biologie Clinique. 2000 ; 4(58) : 431-7.

5. D'Alessandro E: Prévenir le risque infectieux à l'hôpital ? Réflexions anthropologiques autour des pratiques d'hygiène hospitalière dans un service de médecine au Niger. Revue internationale francophone d'anthropologie de la santé. $\quad 2012, \quad 4$. doi.org/10.4000/anthropologiesante.835

6. Hygis $\mathbf{N}$ : Hygiène hospitalière. Presse universitaire de Lyon. Collection Azay 1998.

7. Gabriel G, Lessard-Hébert $\mathbf{M}$ : La recherche action : Ses fonctions, son fondement et son instrumentalisation. Presse de l'Université de Québec. 1987 ; 125p.

8. COULIBALY BK. Contribution à l'amélioration de la gestion des déchets biomédicaux et l'hygiène des mains au CSCOM-U de Sanoubougou II. Mémoire, med, FMOS, Bamako, FMOS, 2019.

9. SIDIBE, D M. Evaluation de la gestion des déchets biomédicaux et l'hygiène des mains au CSCom-U de Banconi. Mémoire, med, FMOS. Bamako, 2016.

10. Brun-Buisson $\mathbf{C}$ : La friction des mains avec une solution hydro-alcoolique : une méthode 
rapide et efficace pour améliorer l'observance de l'hygiène des mains en milieu de soins. La Lettre de l'infectiologue. 2002 ; 17(6) ; 163-164.

11. Dicko-Traore F, Gire C, Malaty VB, Busutill M, Monnier A S, Brunel V et al : Évaluation des connaissances et des pratiques d'hygiène des mains au niveau de l'unité de néonatologie et soins intensifs du service de médecine infantile et néonatologie de l'hôpital Nord, Marseille. Journal de Pédiatrie et de puériculture. 2011 ; $24(4) ; 172-177$.

12. Mallaret MR, Le Coziffenecker A, Duc DL, Brut A, Veyre M, Chaize $P$ et al : Observance du lavage des mains en milieu hospitalier: analyse de la littérature. Médecine et maladies infectieuses. $1998 ; 28: 285-290$.

13. DERRAJI S, MOUKETOU MAHILA J, BAITE A, CHERRAH Y: L'observance de l'hygiène des mains. Maroc médical. 2013 ; 35(4) ; 286289.

14. Ndiaye M, El Metghari L, Soumah MM, Sow ML: Gestion des déchets biomédicaux au sein de cinq structures hospitalières de Dakar, Sénégal. Bulletin de la Société de pathologie exotique. $2012 ; 105$ : 296-304.

15. Yao-Bathaix M F, Bouglouga O, Okon A J, Bangoura AD, DOFFOU S, Kouamé D H et al : Pratiques du personnel paramédical en milieu hospitalier à Abidjan (Côte d'lvoire) face au risque de contamination du virus de l'hépatite $B$ par des accidents de travail. Journal Africain d'Hépato-Gastroentérologie. $2015 ; 9$; 184-188.
16. Clapeau G, Decroix B, Bakayoko-Ly R, Varenne B, Dosso-Hien D, Marie-Odile D : Enquête sur les méthodes de nettoyage, décontamination, désinfection et stérilisation dans des services dentaires en zone tropicale. Cahiers de santé. $1997 ; 7: 323-9$.

17. Duroy $\mathrm{E}$; Le Coutour $\mathrm{X}$ L'hygiène hospitalière et les étudiants en médecine. Médecine et Maladies Infectieuses. $2010 ; 40: 530-536$.

18. Yao-Bathaix M F, Bouglouga O, Okon A J, Bangoura A D, DOFFOU S, Kouamé D H et al : Pratiques du personnel paramédical en milieu hospitalier à Abidjan (Côte d'Ivoire) face au risque de contamination du virus de l'hépatite $B$ par des accidents de travail. Journal Africain d'Hépato-Gastroentérologie. $2015 ; 9$ :184-188.

19. Bengaly L, Fomba S, Keita M, Traoré AT, Harouna z, Fofana A: Impact de l'usage des gants médicaux sur l'observance de l'hygiène des mains au cours des soins au Centre Hospitalier et Universitaire du Point G de Bamako. Revue Malienne d'Infectiologie et de Microbiologie. $2016 ; 7$ : 33-38.

20. E. Girou : Simplification des mesures d'hygiène dans la prévention des infections nosocomiales. Réanimation. 2006 ; 15 ; 193-197.

21. Chevalier B, Margery J, Wade B, Ka S, Diatta B, Gueye $\mathbf{M}$ et al : Perception du risque nosocomial parmi le personnel hospitalier de l'Hôpital Principal de Dakar. Médecine Tropicale. 2008 ; 68 ; 593-596.

Liste des tableaux et figures :
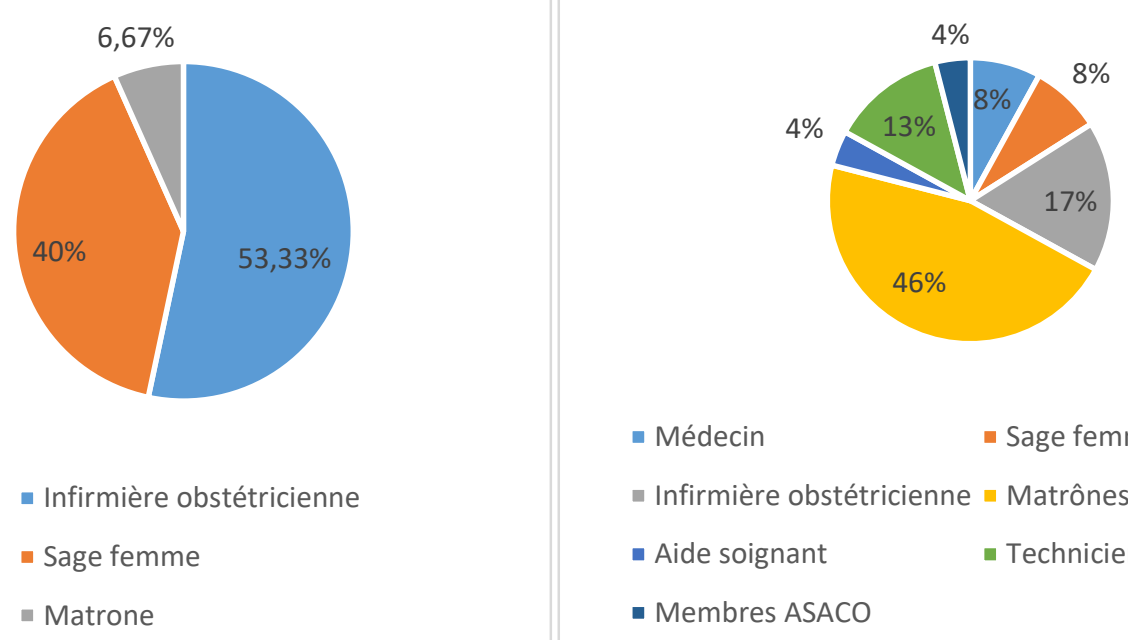

- Médecin $\quad$ - Sage femme

- Infirmière obstétricienne - Matrônes

- Aide soignant - Technicien de surface

- Membres ASACO

Figure I : Personne enquêtée au cours de l'étude

Les infirmières obstétriciennes ont été les plus répresentées à $53,33 \%$ à Banconi tandis que les matrones ont dominée à $46 \%$ à Konobougou. 
Tableau I : Lavage des mains à la prise de service à Banconi

\begin{tabular}{lcc|lcc}
\hline & \multicolumn{2}{c}{ Avant } & & \multicolumn{3}{c}{ Après } \\
\hline Prise de service & Effectif & $\%$ & Prise de service & Effectif & $\%$ \\
Non fait & 11 & 73,33 & Non fait & 01 & 6,67 \\
Fait & 04 & 26,67 & Fait & 14 & 93,33 \\
Total & 15 & 100,00 & Total & 15 & 100,00
\end{tabular}

Les personnels techniques respectant le lavage des mains à la prise de service étaient 93,33\% à l'étape II après l'action contre $26,67 \%$ à l'étape I.

Tableau II : Lavage des mains à la prise de service à Konobougou

\begin{tabular}{lcc|lcc}
\hline \multicolumn{2}{l}{ Avant } & & & Après \\
\hline Prise de service & Effectif & $\%$ & Prise de service & Effectif & $\%$ \\
Non fait & 15 & 65,22 & Non fait & 05 & 21,74 \\
Fait & 08 & 34,78 & Fait & 18 & 78,26 \\
Total & $\mathbf{2 3}$ & $\mathbf{1 0 0 , 0 0}$ & Total & $\mathbf{2 3}$ & $\mathbf{1 0 0 , 0 0}$ \\
\hline
\end{tabular}

Au début de l'enquête nous avons trouvé $34,78 \%$ de personnel respectant le lavage des mains à la prise de service contre $78,26 \%$ à la fin de l'étude.

Tableau III : Préparation d'eau de décontamination à Banconi

\begin{tabular}{lcc|lcc}
\hline \multicolumn{4}{c}{ Avant } & \multicolumn{3}{c}{ Après } \\
\hline Eau de décontamination & Effectif & $\%$ & Eau de décontamination & Effectif & $\%$ \\
Mal préparée & 04 & 26,67 & Mal préparée & 00 & 0,00 \\
Bien préparée & 11 & 73,33 & Bien préparée & 15 & 100,00 \\
Total & 15 & 100,00 & Total & 15 & 100,00
\end{tabular}

En moyenne, onze personnes sur quinze soient 73,33 \% savaient préparer l'eau de décontamination à l'étape I contre $100 \%$ à l'étape II.

Tableau VI : Préparation d'eau de décontamination à Konobougou

\begin{tabular}{lcc|lcc}
\hline \multicolumn{2}{c}{ Avant } & \multicolumn{3}{c}{ Après } \\
\hline Eau de décontamination & Effectif & $\%$ & Eau de décontamination & Effectif & $\%$ \\
Mal préparée & 09 & 45,00 & Mal préparée & 02 & 10,00 \\
Bien préparée & 11 & 55,00 & Bien préparée & 18 & 90,00 \\
Total & 20 & 100,00 & Total & 20 & 100,00 \\
\hline
\end{tabular}

En moyenne, onze personnes sur vingt soient $55 \%$ savaient préparer l'eau de décontamination contre $90 \%$ au terme de l'étude. 